\title{
Heat pretreatment eliminates spurious butyrylcholinesterase enhancement of endotoxin levels in the kinetic chromogenic assay
}

Andrew Brawner ${ }^{1}$, Steven H. Hinrichs ${ }^{1}$, Marilynn A. Larson ${ }^{1}$, Oksana Lockridge ${ }^{2}$

${ }^{1}$ Department of Pathology and Microbiology, ${ }^{2}$ Eppley Institute, University of Nebraska Medical Center, Omaha, NE, 68198 USA

andrew.brawner@unmc.edu

shinrich@unmc.edu

malarson@unmc.edu

olockrid@unmc.edu

Corresponding author:

Oksana Lockridge

985950 Nebraska Medical Center

Omaha, NE 68198

olockrid@unmc.edu

phone 402 559-6032 


\begin{abstract}
The kinetic chromogenic endotoxin assay measures the release of $\mathrm{p}$-nitroaniline from the chromogenic peptide substrate Ac-IEAR-pNA. As part of our project to purify large quantities of human butyrylcholinesterase (HuBChE), we evaluated pure $\mathrm{HuBChE}$ for endotoxin levels. We found that $\mathrm{HuBChE}$ contributed up to $90 \%$ of the yellow p-nitroaniline product in a standard endotoxin assay through the catalytic hydrolysis of Ac-IEAR-pNA with a rate constant of 0.016 $\min ^{-1}$ and a $\mathrm{K}_{\mathrm{m}}$ of $2.9 \mathrm{mM}$ in potassium phosphate buffer $\mathrm{pH} 7.0$ at $24^{\circ} \mathrm{C}$. Thus, endotoxin concentrations for native $\mathrm{BChE}$ are artificially high in the kinetic chromogenic assay.

Destruction of $\mathrm{HuBChE}$ catalytic activity by boiling yields endotoxin concentrations that more accurately reflect the endotoxin concentration in purified HuBChE preparations.
\end{abstract}

Keywords: butyrylcholinesterase, endotoxin, chromogenic substrate, p-nitroacetanilide, amidase

\author{
Abbreviations: \\ HuBChE, human butyrylcholinesterase \\ DMSO, dimethylsulfoxide \\ BTC, butyrylthiocholine \\ 2-NAA, 2-nitroacetanilide \\ Ac-IEAR-pNA, Ac-Ile-Glu-Ala-Arg-pNitroacetanilide \\ LAL, limulus amebocyte lysate \\ $\mathrm{EU} / \mathrm{mL}$, endotoxin units per $\mathrm{mL}$
}




\section{Introduction}

Endotoxins are lipopolysaccharides derived from the cell wall of gram-negative bacteria such as Escherichia coli, Salmonella, and Pseudomonas. Endotoxins injected into animals or humans can cause a systemic inflammatory response, multiple organ failure, septic shock, disseminated intravascular coagulation, and death [1]. Products intended for injection into humans must not deliver more than 5.0 endotoxin units/kg body weight per hour [2]. Rodents can tolerate much higher endotoxin levels than humans [3]. We manufacture pure human butyrylcholinesterase $(\mathrm{HuBChE})$ for use as a nerve agent bioscavenger in animal studies. We measure endotoxin levels in our HuBChE preparations using the kinetic chromogenic assay from Charles River Laboratories.

The Ac-IEAR-pNA peptide is the chromogenic substrate for quantifying endotoxin levels. The kinetic chromogenic endotoxin assay uses a bacterial endotoxin to activate a protease in Limulus Amebocyte Lysate (LAL) that hydrolyzes the colorless substrate Ac-IleGlu-Ala-Arg-pNA, resulting in the production of p-nitroaniline. The time to appearance of the yellow p-nitroaniline product is proportional to the concentration of endotoxin in a sample [4; 5].

HuBChE has aryl acylamidase activity, splitting 2-nitroaniline from 2-nitroacetanilide (2NAA) to produce the yellow 2-nitroaniline product as shown in Figure $1[6 ; 7 ; 8 ; 9]$. Other acetanilides including 3-(acetamido)N,N,N-trimethylanilinium and o-nitrotrifluoroacetanilide are also hydrolyzed by $\mathrm{HuBChE}[7 ; 8]$. 


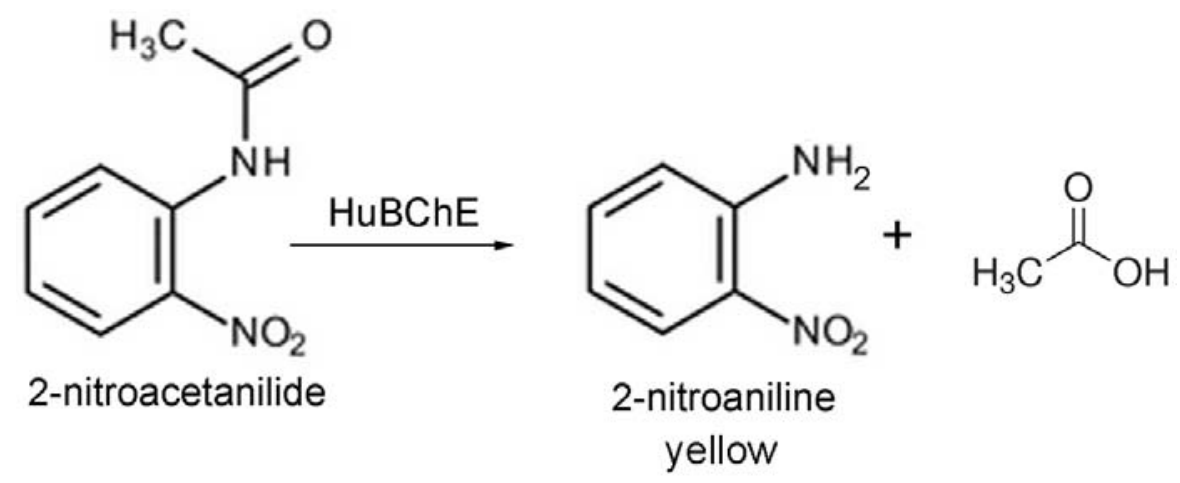

Figure 1. Aryl acylamidase activity of HuBChE.

While testing endotoxin levels in highly concentrated $\mathrm{HuBChE}$ samples, we questioned whether the acylamidase activity of $\mathrm{HuBChE}$ contributed to the apparent endotoxin level in our preparations. If both $\mathrm{HuBChE}$ and the endotoxin-activated LAL hydrolyzed Ac-IEAR-pNA, then the apparent endotoxin concentration in purified $\mathrm{HuBChE}$ preparations would be artificially high.

\section{Materials and Methods}

\subsection{Reagents and materials.}

The Endochrome-K kit was used for the kinetic chromogenic LAL assay of endotoxin levels (Charles River cat\# R1708K). This kit contains the E. coli strain 055:B5 endotoxin standard, LAL reagent water, and lyophilized LAL with chromogenic substrate. Additional LAL was purchased from Charles River (catalog \# R1710K) as needed. The chromogenic substrate Ac-IEAR-pNA peptide (MW 649.7) was obtained from bioWorld (Dublin, OH, catalog \# 21970008-1) and 2-nitroacetanilide was obtained from Lancaster Synthesis Inc. (Pelham, NH, catalog \# 16278). Human BChE (accession \# P06276) was purified from Cohn fraction IV-4 by 
the authors. Hupresin is a new affinity gel for purification of BChE [10]. Hupresin was synthesized by Emilie David at the University of Rouen in France (emilie.david@insa-rouen.fr). The ligand is a small, custom synthesized molecule called huprine 19. The affinity gel was produced by coupling the amine group of huprine 19 to carboxyl groups in ECH-Sepharose 4B (GE Healthcare, catalog \# 17-0571) using carbodiimide. The ECH-Sepharose 4B has a spacer arm of 6-aminohexanoic acid at a density of 12-16 $\mu$ moles carboxyl groups $/ \mathrm{mL}$ of drained gel.

\subsection{Human butyrylcholinesterase.}

Plasma-derived HuBChE was purified from Cohn fraction IV-4 paste by anion exchange chromatography at $\mathrm{pH} 4.5$, followed by affinity chromatography on a hupresin affinity gel [10]. The highly purified HuBChE consisted of tetramers with a molecular weight of $340,000 \mathrm{Da}$. The protein concentration of $\mathrm{HuBChE}$ was calculated from its activity with butyrylthiocholine, using a specific activity of 500 units/mg for BChE purified from frozen Cohn paste.

Activity assays were performed after serially diluting HuBChE into $1 \mathrm{mg} / \mathrm{mL}$ human albumin in phosphate buffered saline to an activity of 5-10 units/mL. HuBChE stock solutions diluted with $1 \mathrm{mg} / \mathrm{mL}$ albumin had 10\% higher activity compared to HuBChE diluted in buffer without albumin. Albumin minimized adsorption of $\mathrm{HuBChE}$ to the walls of the tubes. The activity of a $10 \mu \mathrm{L}$ aliquot of the diluted $\mathrm{HuBChE}$ was assessed in $2 \mathrm{~mL}$ of $0.1 \mathrm{M}$ potassium phosphate pH 7.0 containing $1.0 \mathrm{mM}$ butyrylthiocholine iodide and $0.5 \mathrm{mM}$ 5,5'-dithiobis(2nitrobenzoic acid) at $25^{\circ} \mathrm{C}$. The increase in absorbance at $412 \mathrm{~nm}$ was recorded in a Gilford spectrophotometer interfaced to MacLab. The slope for the time interval 10 to 40 seconds was used to calculate activity using the extinction coefficient $13,600 \mathrm{M}^{-1} \mathrm{~cm}^{-1}$ [11]. Units of activity are defined as $\mu$ moles butyrylthiocholine hydrolyzed per min. 
2.3. Determination of $K_{m}$ and $k_{c a t}$ values for Ac-IEAR-pNA.

A $16 \mathrm{mM}$ stock solution of Ac-IEAR-pNA was prepared by dissolving $0.014 \mathrm{~g}$ in 1.35 $\mathrm{mL}$ of $0.05 \mathrm{M}$ potassium phosphate $\mathrm{pH}$ 7.0. Wells in a 96-well microplate received $90 \mu \mathrm{L}$ of AcIEAR-pNA at different dilutions in buffer and $10 \mu \mathrm{L}$ BChE so that the final Ac-IEAR-pNA concentrations ranged from 1 to $12 \mathrm{mM}$ in a total volume of $100 \mu \mathrm{L}$. The BChE stock solution with an activity of 75,000 units $/ \mathrm{mL}(150 \mathrm{mg} / \mathrm{mL})$ delivered $17.6 \times 10^{-9}$ moles of BChE active sites per well. The absorbance increase at $415 \mathrm{~nm}$ was recorded in a BioTek Synergy H1 Hybrid microplate reader for $60 \mathrm{~min}$ at $24^{\circ} \mathrm{C}$. The rate of hydrolysis of Ac-IEAR-pNA at $415 \mathrm{~nm}$ was calculated using the extinction coefficient $8000 \mathrm{M}^{-1} \mathrm{~cm}^{-1}$ for p-nitroaniline [12] and a pathlength of $0.3 \mathrm{~cm}$ for $100 \mu \mathrm{L}$ sample in a $96-$ well plate.

\subsection{Determination of $K_{m}$ and $k_{\text {cat }}$ values for 2-nitroacetanilide.}

A $0.75 \mathrm{M}$ stock solution of 2-NAA was prepared by dissolving $0.027 \mathrm{~g}$ of 2 -NAA in 0.2 $\mathrm{mL}$ dimethylsulfoxide (DMSO). The solution was diluted to $9.36 \mathrm{~mL}$ with $0.05 \mathrm{M}$ potassium phosphate $\mathrm{pH} 7.0$ to make a $16 \mathrm{mM}$ stock solution containing 2\% DMSO. The $16 \mathrm{mM}$ stock solution was further diluted with $0.05 \mathrm{M}$ potassium phosphate $\mathrm{pH} 7.0$ containing $2 \%$ DMSO to make 2-NAA solutions ranging in concentration from 2 to $15.2 \mathrm{mM}$. Each well in a 96-well plate received $190 \mu \mathrm{L}$ of $2-\mathrm{NAA}$ and $10 \mu \mathrm{L}$ of $\mathrm{BChE}$ (550 units/mL in phosphate buffered saline and $1.5 \mathrm{mM}$ sodium azide) so that the quantity of BChE active sites in each well was $0.13 \times 10^{-9}$ moles (5.5 units of activity). The absorbance increase at $430 \mathrm{~nm}$ was recorded in a BioTek Synergy $\mathrm{H} 1$ hybrid microplate reader for $10 \mathrm{~min}$ at $24^{\circ} \mathrm{C}$. The rate of hydrolysis of 2-NAA at 
$430 \mathrm{~nm}$ was calculated using the extinction coefficient $4000 \mathrm{M}^{-1} \mathrm{~cm}^{-1}$ for 2-nitroaniline [13] and a pathlength of $0.5 \mathrm{~cm}$ for $200 \mu \mathrm{L}$ sample in a 96-well plate.

\subsection{Endotoxin levels in native and heat-denatured HuBChE.}

A pure $\mathrm{HuBChE}$ preparation in $52 \mathrm{mM}$ L-His $\mathrm{pH} 6.0$ with an activity of 20,000 units $/ \mathrm{mL}$ $(40 \mathrm{mg} / \mathrm{mL})$ was serially diluted with endotoxin-free water to evaluate the effect of $\mathrm{BChE}$ amidase activity on the apparent endotoxin levels in the kinetic chromogenic LAL assay. A second set of serially diluted samples was prepared from heat-denatured $\mathrm{HuBChE}$ that had lost all activity with the substrate butyrylthiocholine. The heat-denatured HuBChE samples were prepared by heating a $0.5 \mathrm{~mL}$ aliquot of $\mathrm{HuBChE}(40 \mathrm{mg} / \mathrm{mL})$ in a boiling water bath for $3 \mathrm{~min}$ and serially diluting the denatured $\mathrm{HuBChE}$ with LAL water. Wells of a sterile 96-well plate received $100 \mu \mathrm{L}$ of HuBChE. The hydrolysis reactions were initiated by adding $100 \mu \mathrm{L}$ of the LAL reagent, which contained the chromogenic substrate, to each well after the temperature in the BioTek Synergy H1 hybrid microplate reader had reached $37^{\circ} \mathrm{C}$. The increase in absorbance at $405 \mathrm{~nm}$ was recorded for 1.5 hours. Serial dilutions of the endotoxin standard yielded a standard curve for calculating the endotoxin concentrations in the BChE samples. Data were calculated with Gen5 2.01 Data Analysis Software. The assay is based on the time it takes to see an absorbance increase of 0.2 at $405 \mathrm{~nm}$. The study was repeated four times for a total of 16 replicates for each BChE dilution.

\section{Results}

\subsection{Hydrolysis of Ac-IEAR-pNA peptide by HuBChE.}

The chromogenic substrate Ac-IEAR-pNA was cleaved by $\mathrm{HuBChE}$ to produce the yellow p-nitroaniline product detected at $415 \mathrm{~nm}$ indicated in Figure 2. A control reaction 
containing $1 \mathrm{mM}$ chromogenic peptide, but no $\mathrm{HuBChE}$, showed no change in absorbance during the $1 \mathrm{~h}$ measurement period. This result demonstrated that HuBChE hydrolyzes the amide bond that links p-nitroaniline to arginine in the peptide Ac-IleGluAlaArg-pNA.

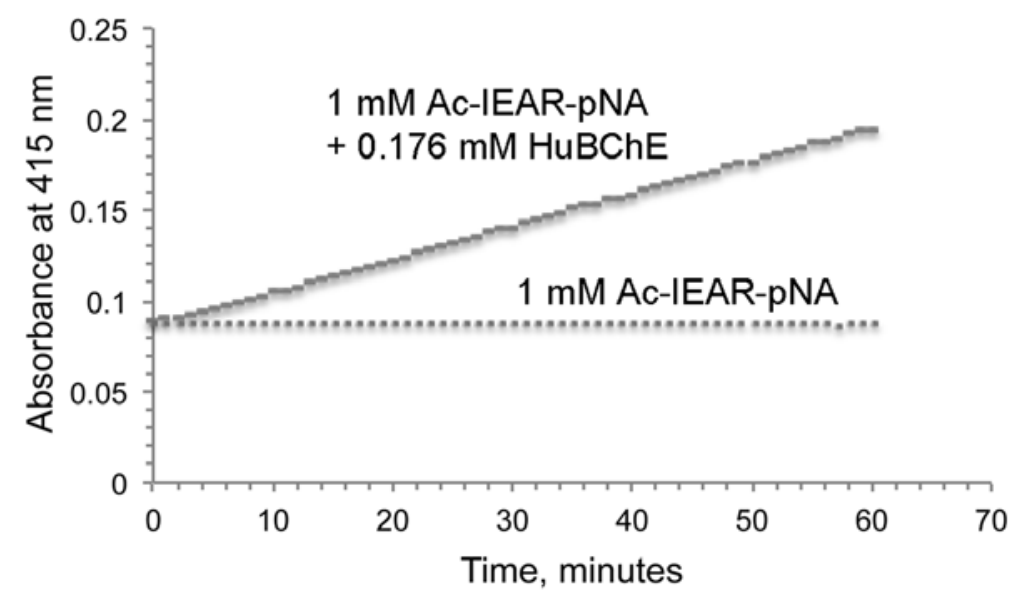

Figure 2. Hydrolysis of Ac-IEAR-pNA by HuBChE. p-Nitroaniline was released from $1 \mathrm{mM}$ Ac-IEAR-pNA in $0.05 \mathrm{M}$ potassium phosphate $\mathrm{pH} 7.0$ by the amidase activity of 750 units $(1.5$ $\mathrm{mg}$ ) of $\mathrm{HuBChE}$ in $100 \mu \mathrm{L}$ at $24^{\circ} \mathrm{C}$. No hydrolysis of Ac-IEAR-pNA was observed in the absence of HuBChE.

3.2. $K_{m}$ and $k_{c a t}$ values for Ac-IEAR-pNA hydrolysis by HuBChE.

The rate of hydrolysis of the chromogenic substrate increased 3-fold when the concentration of Ac-IEAR-pNA was increased from $1 \mathrm{mM}$ to $8 \mathrm{mM}$, as shown in Figure $3 \mathrm{~A}$. No further increase in rate was observed with Ac-IEAR-pNA concentrations higher than $8 \mathrm{mM}$. 

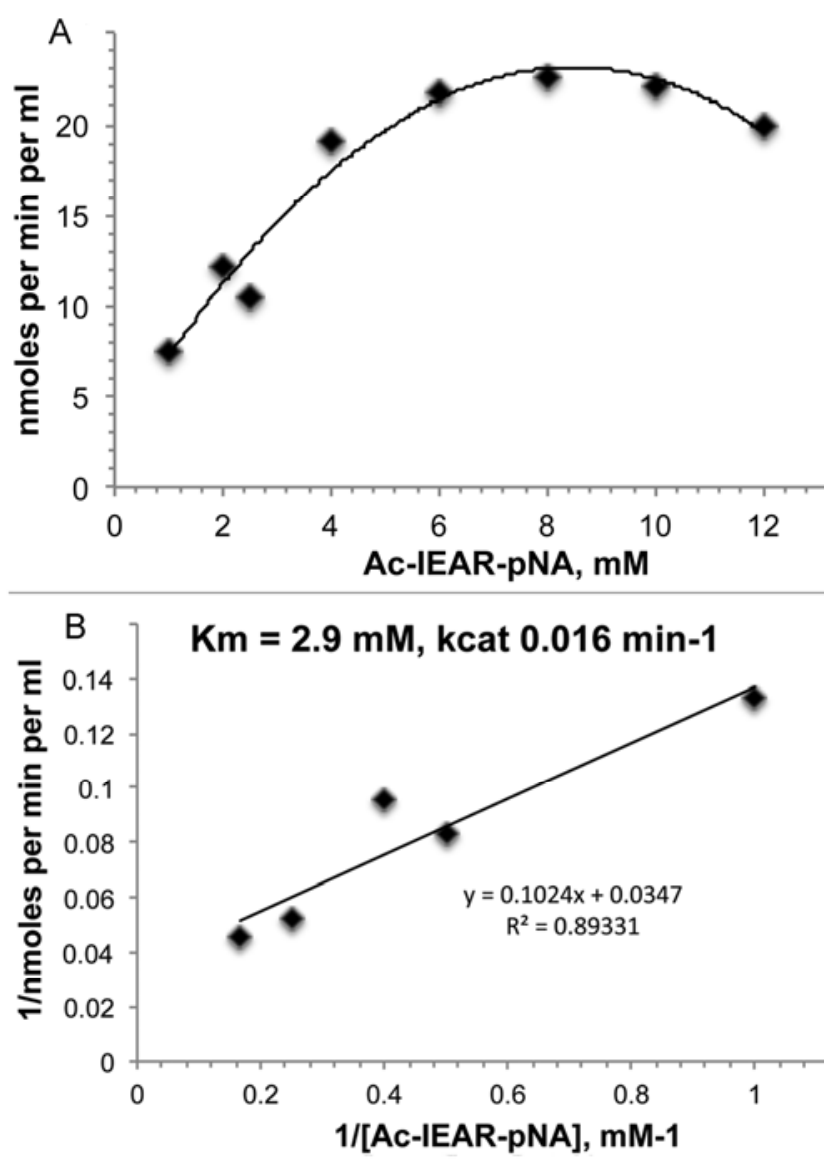

Figure 3. A) Rate of hydrolysis of Ac-IEAR-pNA by HuBChE. The $100 \mu \mathrm{L}$ reaction volume contained 0.176 mM HuBChE and 1-12 mM Ac-IEAR-pNA in 0.05 M potassium phosphate $\mathrm{pH}$ 7.0. The reaction was monitored for 60 minutes at $24^{\circ} \mathrm{C}$. B) Determination of $\mathrm{K}_{\mathrm{m}}$ and $\mathrm{k}_{\mathrm{cat}}$ values for the hydrolysis of Ac-IEAR-pNA by HuBChE in $0.05 \mathrm{M}$ potassium phosphate $\mathrm{pH} 7.0$ at $24^{\circ} \mathrm{C}$, using 750 units $(1.5 \mathrm{mg})$ of $\mathrm{HuBChE}$ per $100 \mu \mathrm{L}$ reaction.

The Lineweaver-Burk plot in Figure 3B yielded a $\mathrm{K}_{\mathrm{m}}$ value of $2.9 \mathrm{mM}$ and a $\mathrm{k}_{\text {cat }}$ value of $0.016 \mathrm{~min}^{-1}$ for the hydrolysis of Ac-IEAR-pNA by HuBChE. 
3.3. $K_{m}$ and $k_{\text {cat }}$ values for 2-NAA hydrolysis by HuBChE.

In the present study we confirmed the work of others that HuBChE hydrolyzes 2nitroacetanilide $[6 ; 7 ; 8]$. Figure 4 shows the Lineweaver-Burk plot from which we derived the $\mathrm{K}_{\mathrm{m}}$ and $\mathrm{k}_{\mathrm{cat}}$ values for the hydrolysis of 2-NAA by HuBChE.

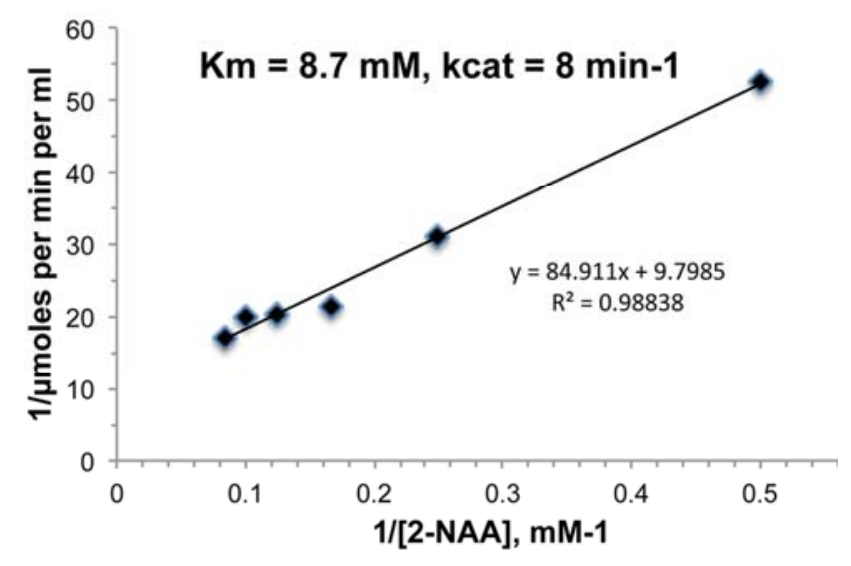

Figure 4. Determination of $\mathrm{K}_{\mathrm{m}}$ and $\mathrm{k}_{\mathrm{cat}}$ values for 2-NAA hydrolysis by $\mathrm{HuBChE}$ in $0.05 \mathrm{M}$ potassium phosphate $\mathrm{pH} 7.0$ at $24^{\circ} \mathrm{C}$, using 5.5 units $(0.011 \mathrm{mg})$ of $\mathrm{HuBChE}$ per $200 \mu \mathrm{L}$ reaction.

\subsection{Comparison of endotoxin levels in native and heat-denatured HuBChE.}

Solutions of native $\mathrm{HuBChE}$ at concentrations ranging from 20 units $/ \mathrm{mL}(0.04 \mathrm{mg} / \mathrm{mL})$ to 20,000 units $/ \mathrm{mL}$ ( $40 \mathrm{mg} / \mathrm{mL}$ ) were evaluated for endotoxin levels, using the kinetic chromogenic assay. These results demonstrated that an increase in $\mathrm{HuBChE}$ concentration coincided with an increase in the apparent endotoxin level for $\mathrm{HuBChE}$ concentrations ranging from 20 to 2000 units $/ \mathrm{mL}$. However, the highest $\mathrm{HuBChE}$ concentration, 20,000 units $/ \mathrm{mL}$, had a suppressed endotoxin level. This means the high protein concentration or the buffer $(52 \mathrm{mM}$ L-His $\mathrm{pH} 6.0$ ) in the 20,000 units $/ \mathrm{mL}$ HuBChE, inhibited LAL activity. The LAL reaction is enzyme mediated and has an optimal $\mathrm{pH}$ range and specific salt and divalent cation requirements 
that may have been altered by the 20,000 units/mL sample. As shown in Table 1 , boiling the $\mathrm{HuBChE}$ to denature its amidase activity reduced the observed endotoxin concentration up to 90\%. The method selected for denaturing HuBChE was boiling, since boiling does not destroy endotoxins nor introduce inhibitory chemicals that might affect the function of the LAL proteases, which are essential for quantifying endotoxin levels in the kinetic chromogenic assay.

Table 1. Comparison of endotoxin values for native and heat-denatured HuBChE.

\begin{tabular}{|c|c|c|c|}
\hline $\begin{array}{c}\text { Activity } \\
\text { (units/mL) }\end{array}$ & Native HuBChE & Boiled HuBChE & \% Decrease \\
\hline 20,000 & $10.3 \pm 8.3$ & $1.1 \pm 0.4$ & 90 \\
\hline 2,000 & $22.7 \pm 25.9$ & $6.6 \pm 3.6$ & 70 \\
\hline 200 & $3.5 \pm 3.0$ & $1.6 \pm 0.5$ & 54 \\
\hline 20 & $1.3 \pm 0.7$ & $0.18 \pm 0.08$ & 86 \\
\hline
\end{tabular}

\section{Discussion}

4.1. Interference with the kinetic chromogenic assay for endotoxin.

Native HuBChE (0.04 to $40 \mathrm{mg} / \mathrm{mL})$ interferes with the kinetic chromogenic assay for determining endotoxin levels by introducing a non-specific overestimation of the endotoxin concentration. Artificial enhancement of the endotoxin level could cause a HuBChE preparation to fail FDA standards for product release. Denaturing the HuBChE in a boiling water bath for 3 min before testing the $\mathrm{HuBChE}$ in the kinetic chromogenic assay solved the problem of 
endotoxin enhancement by native HuBChE. Boiling selectively inactivates $\mathrm{HuBChE}$ activity, but does not destroy endotoxin. Endotoxins survive $30 \mathrm{~min}$ at $100^{\circ} \mathrm{C}[14]$.

\subsection{HuBChE hydrolyzes LAL chromogenic substrate.}

A summary is presented in Table 2 comparing the $\mathrm{K}_{\mathrm{m}}$ and $\mathrm{k}_{\text {cat }}$ values for $\mathrm{HuBChE}$ hydrolysis of the chromogenic substrate Ac-IEAR-pNA to 2-nitroacetanilide (2-NAA) and butyrylthiocholine (BTC). The apparent affinity constants $\left(\mathrm{K}_{\mathrm{m}}\right)$ for both Ac-IEAR-pNA and 2NAA are in the mM range. Amidolysis of peptide Ac-IEAR-pNA proceeds at a 500-fold slower rate $\left(k_{c a t} 0.016\right.$ min-1) compared to the rate obtained for 2-nitroacetanilide $\left(k_{c a t} 8 \min ^{-1}\right)$. In contrast, esterolysis of butyrylthiocholine is $1,500,000$-fold faster $\left(\mathrm{k}_{\mathrm{cat}} 24,000 \mathrm{~min}^{-1}\right)$. Despite the relatively slow rate of hydrolysis of the LAL chromogenic substrate by HuBChE, the rate is comparable to that by the endotoxin-induced LAL proteases. The consequence is that native $\mathrm{HuBChE}$ preparations can contribute up to $90 \%$ of the yellow product leading to overestimation of the endotoxin concentration in the kinetic chromogenic assay. The approach described here effectively eliminates the artificial enhancement of endotoxin levels by $\mathrm{HuBChE}$ and allows for accurate determination of the endotoxin concentration in $\mathrm{HuBChE}$ samples. 
Table 2. Affinity and rate constants for the hydrolysis of acylamides and ester by HuBChE.

\begin{tabular}{|c|c|c|c|c|c|c|}
\hline Substrate & $\begin{array}{c}\mathrm{K}_{\mathrm{m}} \\
(\mathrm{mM})\end{array}$ & $\begin{array}{c}\text { kcat } \\
\left(\min ^{-1}\right)\end{array}$ & Buffer & pH & ${ }^{\circ} \mathbf{C}$ & Reference \\
\hline $\begin{array}{c}\text { Ac-IEAR- } \\
\text { pNA }\end{array}$ & 2.9 & 0.016 & $0.05 \mathrm{M} \mathrm{K}$ phosphate & 7 & 24 & This study \\
\hline 2-NAA & 8.7 & 8 & $\begin{array}{c}0.05 \mathrm{M} \mathrm{K} \text { phosphate, } \\
2 \% \mathrm{DMSO}\end{array}$ & 7 & 24 & This study \\
\hline 2-NAA & 0.6 & 3 & $\begin{array}{c}0.05 \mathrm{M} \text { TrisCl } 1.6 \% \\
\text { acetonitrile }\end{array}$ & 8 & 23 & [8] \\
\hline 2-NAA & $>3$ & $>13$ & $\begin{array}{c}\text { 0.1 M K phosphate } \\
\text { 5\% methanol }\end{array}$ & 7 & 25 & {$[7]$} \\
\hline BTC & 0.018 & 24,000 & $0.1 \mathrm{M} \mathrm{K}$ phosphate & 7 & 25 & [15] \\
\hline
\end{tabular}

\section{Acknowledgment}

This research was supported in part by contract HDTRA 1-14-1-0056 to SHH from the Defense

Threat Reduction Agency. The views expressed in this article are those of the authors and do not reflect the official policy of the United States Department of Defense, United States Government, or any United States Federal agency.

\section{References}

[1] S.M. Opal, The host response to endotoxin, antilipopolysaccharide strategies, and the management of severe sepsis. Int J Med Microbiol 297 (2007) 365-77. 
[2] J. Cooper, FDA withdraws LAL test Guideline. Endosafe Times Charles River: Endotoxin and microbial detection newsletter. 15 (2011).

[3] H.S. Warren, C. Fitting, E. Hoff, M. Adib-Conquy, L. Beasley-Topliffe, B. Tesini, X. Liang, C. Valentine, J. Hellman, D. Hayden, J.M. Cavaillon, Resilience to bacterial infection: difference between species could be due to proteins in serum. J Infect Dis 201 (2010) 223-32.

[4] R.N. Berzofsky, Endotoxin Detection in Pharmaceuticals and Medical Devices with Kinetic-QCL, a Kinetic-Quantitative Chromogenic Limulus Amebocyte Lysate Assay. ALTEX $12(1995)$ 93-97.

[5] G.N. Keller, R.N. Berzofsky, R. Burt, J. Stefka, A. Traynor, C. Link, A. LeFever, The determination of endotoxin in the finished cellular product. Cytotherapy 1 (1999) 423-8. [6] L. Jaganathan, R. Boopathy, A direct method to visualise the aryl acylamidase activity on cholinesterases in polyacrylamide gels. BMC Biochem 1 (2000) 3.

[7] P. Masson, M.T. Froment, E. Gillon, F. Nachon, S. Darvesh, L.M. Schopfer, Kinetic analysis of butyrylcholinesterase-catalyzed hydrolysis of acetanilides. Biochim Biophys Acta 1774 (2007) 1139-47.

[8] S. Darvesh, R.S. McDonald, K.V. Darvesh, D. Mataija, S. Mothana, H. Cook, K.M. Carneiro, N. Richard, R. Walsh, E. Martin, On the active site for hydrolysis of aryl amides and choline esters by human cholinesterases. Bioorg Med Chem 14 (2006) 4586-99.

[9] M.F. Montenegro, M.T. Moral-Naranjo, M. Paez de la Cadena, F.J. Campoy, E. MunozDelgado, C.J. Vidal, The level of aryl acylamidase activity displayed by human butyrylcholinesterase depends on its molecular distribution. Chem Biol Interact 175 (2008) 336-9. 
[10] X. Brazzolotto, M. Wandhammer, C. Ronco, M. Trovaslet, L. Jean, O. Lockridge, P.Y. Renard, F. Nachon, Human butyrylcholinesterase produced in insect cells: huprine-based affinity purification and crystal structure. FEBS J 279 (2012) 2905-16.

[11] G.L. Ellman, K.D. Courtney, V. Andres, Jr., R.M. Feather-Stone, A new and rapid colorimetric determination of acetylcholinesterase activity. Biochem. Pharmacol. 7 (1961) 88-95.

[12] R. Lottenberg, C.M. Jackson, Solution composition dependent variation in extinction coefficients for p-nitroaniline. Biochim Biophys Acta 742 (1983) 558-64.

[13] R.E. Hoagland, G. Graf, Nitroacetanilides as chromogenic substrates for assaying deacetylating activity: the isolation and partial purification of aryl acylamidases from erepsin and tulip. Enzymologia 41 (1971) 313-9.

[14] T. Miyamoto, S. Okano, N. Kasai, Inactivation of Escherichia coli endotoxin by soft hydrothermal processing. Appl Environ Microbiol 75 (2009) 5058-63.

[15] P. Masson, P. Legrand, C.F. Bartels, M.T. Froment, L.M. Schopfer, O. Lockridge, Role of aspartate 70 and tryptophan 82 in binding of succinyldithiocholine to human butyrylcholinesterase. Biochemistry 36 (1997) 2266-77. 


\subsection{Reagents and materials.}

The Endochrome-K kit was used for the kinetic chromogenic LAL assay of endotoxin levels (Charles River cat\# R1708K). This kit contains the E. coli strain 055:B5 endotoxin standard, LAL reagent water, and lyophilized LAL with chromogenic substrate. Additional LAL was purchased from Charles River (catalog \# R1710K) as needed. The chromogenic substrate Ac-IEAR-pNA peptide (MW 649.7) was obtained from bioWorld (Dublin, OH, catalog \# 21970008-1) and 2-nitroacetanilide was obtained from Lancaster Synthesis Inc. (Pelham, NH, catalog \# 16278). Human BChE (accession \# P06276) was purified from Cohn fraction IV-4 by 
containing $1 \mathrm{mM}$ chromogenic peptide, but no HuBChE, showed no change in absorbance during the $1 \mathrm{~h}$ measurement period. This result demonstrated that HuBChE hydrolyzes the amide bond that links p-nitroaniline to arginine in the peptide Ac-IleGluAlaArg-pNA.

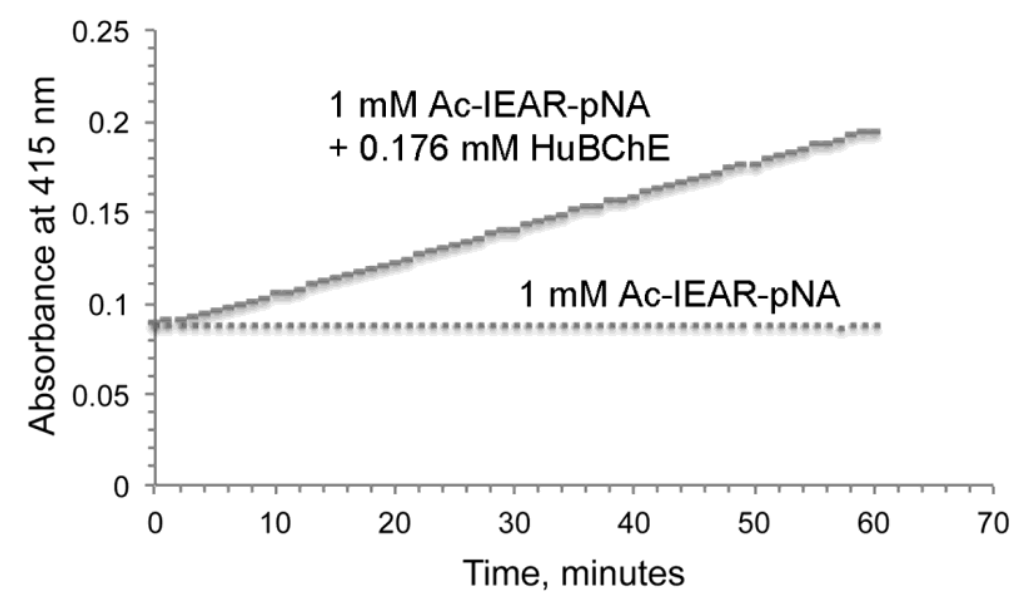

Figure 2. Hydrolysis of Ac-IEAR-pNA by HuBChE. p-Nitroaniline was released from $1 \mathrm{mM}$ Ac-IEAR-pNA in $0.05 \mathrm{M}$ potassium phosphate $\mathrm{pH} 7.0$ by the amidase activity of 750 units $(1.5$ $\mathrm{mg}$ ) of $\mathrm{HuBChE}$ in $100 \mu \mathrm{L}$ at $24^{\circ} \mathrm{C}$. No hydrolysis of Ac-IEAR-pNA was observed in the absence of HuBChE.

3.2. $K_{m}$ and $k_{\text {cat }}$ values for Ac-IEAR-pNA hydrolysis by HuBChE.

The rate of hydrolysis of the chromogenic substrate increased 3-fold when the concentration of Ac-IEAR-pNA was increased from $1 \mathrm{mM}$ to $8 \mathrm{mM}$, as shown in Figure $3 \mathrm{~A}$. No further increase in rate was observed with Ac-IEAR-pNA concentrations higher than $8 \mathrm{mM}$. 

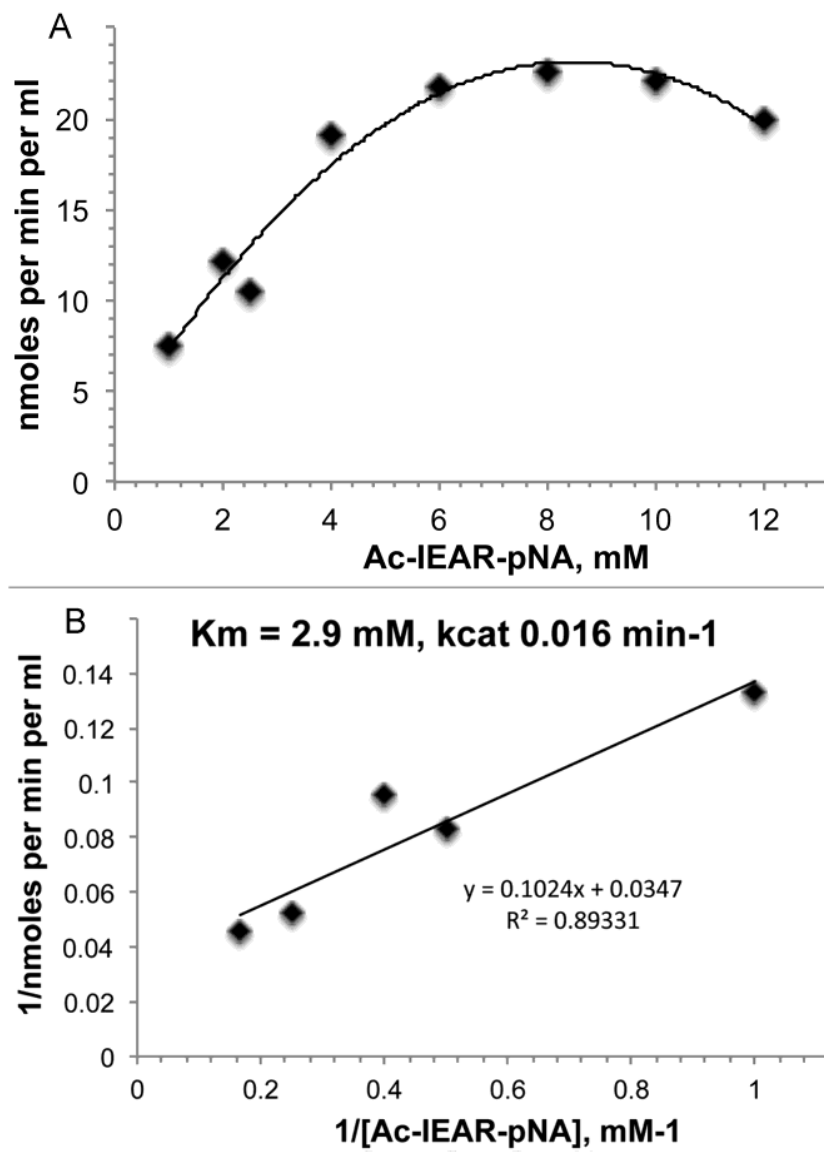

Figure 3. A) Rate of hydrolysis of Ac-IEAR-pNA by HuBChE. The $100 \mu \mathrm{L}$ reaction volume contained $0.176 \mathrm{mM}$ HuBChE and 1-12 mM Ac-IEAR-pNA in 0.05 M potassium phosphate $\mathrm{pH}$ 7.0. The reaction was monitored for 60 minutes at $24^{\circ} \mathrm{C}$. B) Determination of $\mathrm{K}_{\mathrm{m}}$ and $\mathrm{k}_{\mathrm{cat}}$ values for the hydrolysis of Ac-IEAR-pNA by HuBChE in $0.05 \mathrm{M}$ potassium phosphate $\mathrm{pH} 7.0$ at $24^{\circ} \mathrm{C}$, using 750 units $(1.5 \mathrm{mg})$ of $\mathrm{HuBChE}$ per $100 \mu \mathrm{L}$ reaction.

The Lineweaver-Burk plot in Figure 3B yielded a $\mathrm{K}_{\mathrm{m}}$ value of $2.9 \mathrm{mM}$ and a $\mathrm{k}_{\text {cat }}$ value of $0.016 \mathrm{~min}^{-1}$ for the hydrolysis of Ac-IEAR-pNA by HuBChE. 
3.3. $K_{m}$ and $k_{\text {cat }}$ values for 2-NAA hydrolysis by HuBChE.

In the present study we confirmed the work of others that $\mathrm{HuBChE}$ hydrolyzes 2-

nitroacetanilide $[6 ; 7 ; 8]$. Figure 4 shows the Lineweaver-Burk plot from which we derived the $\mathrm{K}_{\mathrm{m}}$ and $\mathrm{k}_{\mathrm{cat}}$ values for the hydrolysis of 2-NAA by HuBChE.

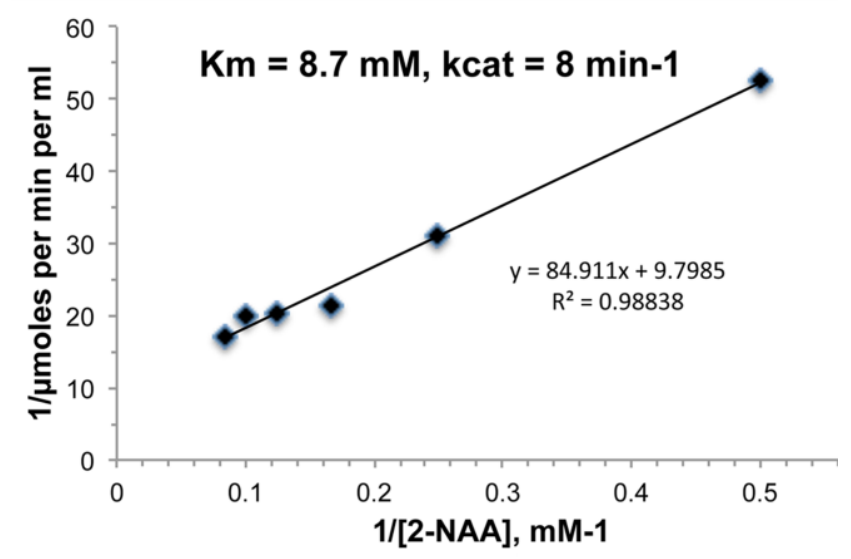

Figure 4. Determination of $\mathrm{K}_{\mathrm{m}}$ and $\mathrm{k}_{\mathrm{cat}}$ values for 2-NAA hydrolysis by HuBChE in $0.05 \mathrm{M}$ potassium phosphate $\mathrm{pH} 7.0$ at $24^{\circ} \mathrm{C}$, using 5.5 units $(0.011 \mathrm{mg})$ of $\mathrm{HuBChE}$ per $200 \mu \mathrm{L}$ reaction.

\subsection{Comparison of endotoxin levels in native and heat-denatured HuBChE.}

Solutions of native $\mathrm{HuBChE}$ at concentrations ranging from $20 \mathrm{units} / \mathrm{mL}(0.04 \mathrm{mg} / \mathrm{mL})$ to 20,000 units $/ \mathrm{mL}(40 \mathrm{mg} / \mathrm{mL}$ ) were evaluated for endotoxin levels, using the kinetic chromogenic assay. These results demonstrated that an increase in $\mathrm{HuBChE}$ concentration coincided with an increase in the apparent endotoxin level for $\mathrm{HuBChE}$ concentrations ranging from 20 to 2000 units $/ \mathrm{mL}$. However, the highest $\mathrm{HuBChE}$ concentration, 20,000 units $/ \mathrm{mL}$, had a suppressed endotoxin level. This means the high protein concentration or the buffer $(52 \mathrm{mM}$ L-His $\mathrm{pH}$ 6.0) in the 20,000 units/mL HuBChE, inhibited LAL activity. The LAL reaction is enzyme mediated and has an optimal $\mathrm{pH}$ range and specific salt and divalent cation requirements 